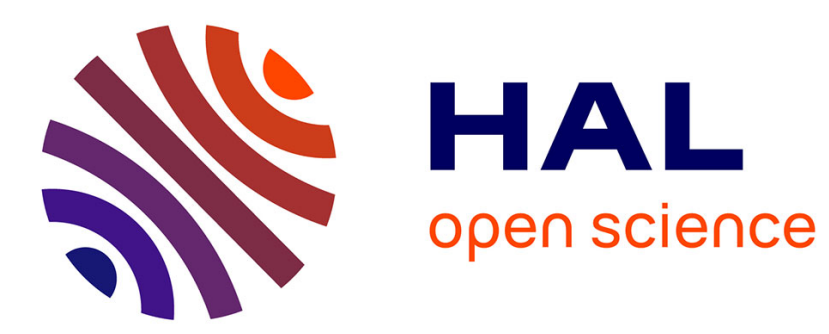

\title{
Alliage de surface et équilibre local dans le système $\mathrm{Pd} / \mathrm{Cu}(111)$ \\ C. Gallis, B. Legrand, G. Tréglia
}

\section{To cite this version:}

C. Gallis, B. Legrand, G. Tréglia. Alliage de surface et équilibre local dans le système $\mathrm{Pd} / \mathrm{Cu}(111)$. Journal de Physique IV Proceedings, 1996, 06 (C7), pp.C7-155-C7-158. 10.1051/jp4:1996718 jpa00254506

\section{HAL Id: jpa-00254506 https://hal.science/jpa-00254506}

Submitted on 1 Jan 1996

HAL is a multi-disciplinary open access archive for the deposit and dissemination of scientific research documents, whether they are published or not. The documents may come from teaching and research institutions in France or abroad, or from public or private research centers.
L'archive ouverte pluridisciplinaire HAL, est destinée au dépôt et à la diffusion de documents scientifiques de niveau recherche, publiés ou non, émanant des établissements d'enseignement et de recherche français ou étrangers, des laboratoires publics ou privés. 


\title{
Alliage de surface et équilibre local dans le système $\mathrm{Pd} / \mathrm{Cu}$ (111)
}

\author{
C. Gallis, B. Legrand et G. Tréglia* \\ SRMP/DECM, CEA Saclay, 91191 Gif-sur-Yvette cedex, France \\ * CRMC2-CNRS, Campus de Luminy, Case 913, 13288 Marseille cedex 9, France
}

\begin{abstract}
Résumé : Nous avons étudié les cinétiques de formation et de dissolution d'alliage de surface obtenus lors de dépôt de $\mathrm{Pd} / \mathrm{Cu}$ (111) dans le cadre d'un modèle cinétique (KTBIM). Selon l'épaisseur du dépôt initial, nous mettons en évidence une succession spatio-temporelle d'alliages ordonnés de surface au cours de la cinétique. Le concept d'équilibre local nous permet de relier les profils de concentration obtenus lors de la cinétique à des profils d'équilibre de couches minces supportées. L'extension spatio-temporelle du concept d'équilibre local sera également discutée.
\end{abstract}

\section{INTRODUCTION}

La stabilité thermique des multicouches métalliques et l'interdiffusion lors de la croissance de celles-ci font l'objet de très nombreuses études expérimentales [1]. La modélisation des phénomènes d'interdiffusion sur ces objets d'épaisseur nanométrique, où la surface et les interfaces jouent un grand rôle, requiert un modèle cinétique décrivant correctement à la fois la diffusion en volume et les cinétiques superficielles, telles que les cinétiques de ségrégation de surface par exemple. Ceci nécessite donc la prise en compte de l'ensemble des facteurs énergétiques pilotant les phénomènes d'ordre en volume ainsi que la ségrégation superficielle. Ces multiples exigences expliquent sans doute pourquoi les travaux théoriques sont encore peu développés sur ce thème [2].

Nous étudions dans ce travail l'influence de l'épaisseur du dépôt sur les cinétiques de formation et de dissolution des alliages de surface obtenus par dépôt de $\mathrm{Pd} / \mathrm{Cu}$ (111). Historiquement, c'est un des premiers systèmes sur lequel la formation d'alliage de surface a été observée, les expériences étant initialement consacrées à la face (100), mais s'étendant actuellement aux autres faces [3].

Après avoir rappelé les caractéristiques essentielles du modèle cinétique utilisé (\$2), nous présenterons les cinétiques de dissolution de 1 et 10 monocouches $(\mathrm{MC})$ de $\mathrm{Pd}$ déposées sur $\mathrm{Cu}(111)(\S 3)$ puis nous les analyserons en développant la notion d'équilibre local (§ 4).

\section{MODELE CINETIQUE: le KTBIM (Kinetic Tight-Binding Ising Model)}

Afin de décrire les cinétiques de dissolution, nous avons utilisé le modèle KTBIM largement détaillé dans la référence [2]. Le cristal est décrit comme un empilement de plans atomiques p parallèles à la surface (plan $\mathrm{p}=0$ ), ces plans étant supposés être homogènes đans l'état désordonné et homogènes par sous-réseau dans les états ordonnés. Les fréquences de saut d'un plan à l'autre (et plus généralement d'un sous-réseau à l'autre) à l'instant $t$ dépendent des moteurs énergétiques qui pilotent la ségrégation superficielle et l'ordre en volume, ainsi que des concentrations à l'instant $t$, ceci dans l'approximation de champ moyen [4]. On obtient ainsi l'évolution au cours du temps du profil de concentration $\left(c_{p}^{\alpha}(t)\right)$, où $c_{p}^{\alpha}(t)$ est la concentration de l'élément déposé sur le sous-réseau $\alpha$ dans le plan p à l'instant $t$.

Quatre sous-réseaux ( $\alpha, \beta, \gamma$ et $\delta$ ) par plan (111) sont nécessaires pour décrire les structures ordonnées d'équilibre ( $\mathrm{L}_{10}, \mathrm{~L}$ ' et $\mathrm{L}_{12}$ ) du diagramme de phase volumique dans l'approximation employée [5] (interaction aux premiers voisins sur réseau rigide CFC en Bragg-Williams). Nous définissons le paramètre d'ordre par plan, $\eta_{p}(t)$, comme $\eta_{p}(t)=\left(c_{p}^{\alpha}(t)+c_{p}^{\beta}(t)-c_{p}^{\gamma}(t)-c_{p}^{\delta}(t)\right) / 2$, respectivement égal à 1 , $x$ (avec $0,5<x<1$ ) et 0,5 pour les phases $L_{10}$, L' et L12 parfaitement ordonnées. 
Les valeurs des différentes quantités énergétiques (contribution de l'effet de tension et de l'effet de taille à l'énergie de ségrégation superficielle et interaction effective d'alliage $\mathrm{V}$ ) et cinétiques (coefficient de diffusion en volume D) sont données dans les références [6,7].

Par souci de généralisation, les températures sont données en $\mathrm{T} / \mathrm{T}_{\mathrm{c}}$ (où $\mathrm{kT} \mathrm{T}_{\mathrm{C}}=2 \mathrm{~V}$ est la température critique en volume à $c=0,5)$, les temps étant portés en $t / t_{0}$ où to est un temps caractéristique de diffusion ( $t_{0}=a^{2} /$ $\mathrm{D}$, avec a paramètre de maille).

\section{RÉSULTATS}

Nous présentons dans cette partie les cinétiques de dissolution pour des dépôts de 1 et $10 \mathrm{MC}$ de $\mathrm{Pd} / \mathrm{Cu}$ (111) à $\mathrm{T} / \mathrm{T}_{\mathrm{C}}=0,82$. A cette température, les phases apparaissant dans le diagramme d'équilibre du modèle sont la phase désordonnée $(c<0,245)$, la phase $\mathrm{L}_{12}(0,256<\mathrm{c}<0,41)$ et la phase $\mathrm{L}_{0}(0,423<\mathrm{c})$, le diagramme étant symétrique par rapport à $\mathrm{c}=0,5$. Un domaine biphasé existe entre chaque domaine monophasé.

\subsection{Dépôt de 1 MC Pd / Cu (111)}

La figure 1 montre la dissolution de $1 \mathrm{MC}$ de $\mathrm{Pd} / \mathrm{Cu}$ au cours du temps. Deux régimes peuvent être clairement différenciés : un régime court présentant des variations rapides des concentrations de surface (co) et du premier plan sous la surface $\left(\mathrm{c}_{1}\right)$ et un régime où les variations de concentration sont plus lentes. La séparation des deux régimes (vers $\mathrm{t} / \mathrm{t} 0=0,1$ ) est caractérisée par le maximum de $\mathrm{c} 1$.

De plus, dans le régime lent, la concentration de palladium à la surface est nettement inférieure à celle des plans sous jacents, le profil de concentration étant oscillant sur les trois premiers plans, puis monotone (et quasiment homogène à cette échelle) sur les plans suivants. Cette demière caractéristique est semblable à ce qui est obtenu dans une diffusion Fickienne (i.e. quand il n'y a aucun facteur énergétique et que la seule force motrice de la dissolution est le gradient de concentration). Nous verrons dans le paragraphe 4 , comment le concept d'équilibre local permet d'interpréter ces observations.

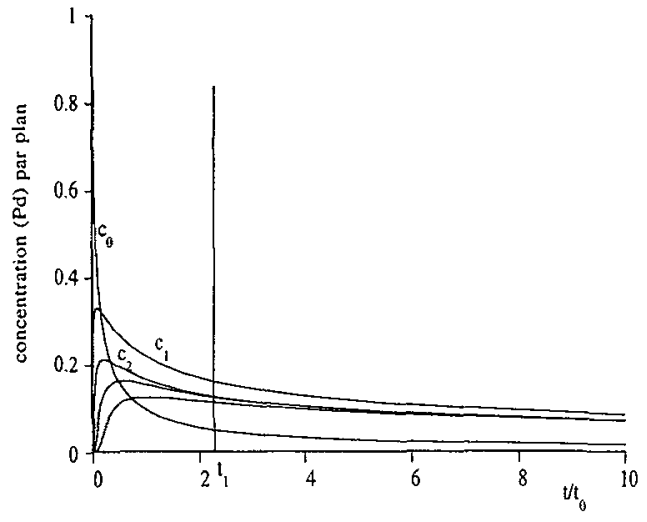

Figure 1: Évolution du profil de concentration au cours du temps $c_{p}(t)$ (pour $\left.0 \leq p \leq 4\right)$ pour la dissolution de $1 \mathrm{MC} \mathrm{Pd} /$ $\mathrm{Cu}(111)$ à $\mathrm{T} / \mathrm{Tc}=0,82$.

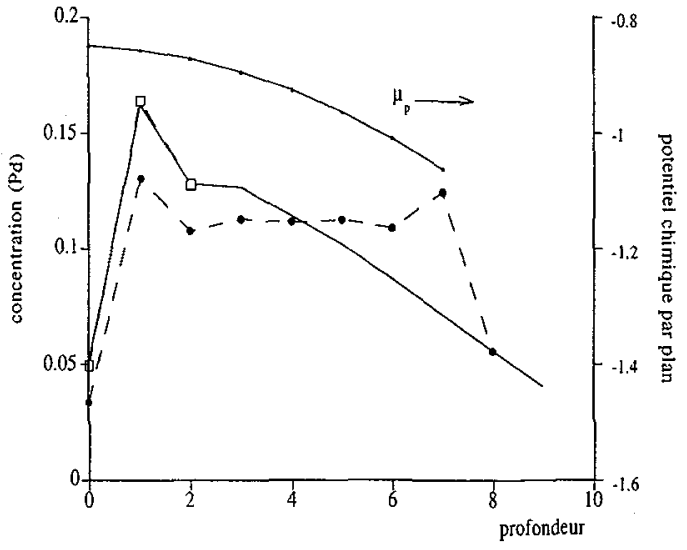

Figure 2: Comparaison des profils de concentration obtenus à l'instant t 1 de la cinétique (ligne continue) et par l'équilibre local (tirets) pour différentes épaisseurs égales à 8 (cercles noir) et 2 (carrés) plans sous la surface, et évolution du potentiel chimique par plan à l'instant $t_{1}$ de la cinétique.

\subsection{Dépôt de 10 MC Pd / Cu (111)}

La dissolution d'une monocouche ne conduit pas à l'apparition de structures ordonnées en surface. A l'inverse, pour un dépôt épais (tendant à la limite vers un couple de diffusion), on s'attend à voir apparaître les phases d'équilibre à partir de l'interface initiale. En volume, l'ordre d'apparition de ces phases et les migrations des parois d'interphase permettant la croissance ou la disparition des phases est un problème encore largement ouvert [8]. Dans le cas d'un dépôt d'épaisseur finie, se rajoute à cela la dissolution progressive des phases formées et l'influence de la surface.

Le schéma de la figure 3 montre l'évolution spatio-temporelle d'un dépôt de $10 \mathrm{MC}$ de $\mathrm{Pd} / \mathrm{Cu}(111)$ à $\mathrm{T} /$ $\mathrm{T}_{\mathrm{C}}=0,82$. Les phases $\mathrm{L} 12$ et L10 se créent à partir de l'interface initiale. Au cours du temps, les phases 
remontent vers la surface, qui voit donc se succéder les phases de moins en moins riches en $\mathrm{Pd}(\mathrm{Pd} 3 \mathrm{Cu}$ puis $\mathrm{PdCu}$ puis $\mathrm{PdCu})$. Enfin cette dernière disparaît pour laisser place à la solution solide $\mathrm{Cu}(\mathrm{Pd})$. On peut se demander quel est l'état d'ordre de la surface lors de ce défilement de phases ordonnées. Ceci est illustré figure 4 où l'évolution temporelle des concentrations par sous-réseau est donnée pour le plan de surface. On voit bien les trois phases ( $\mathrm{L1}_{2}, \mathrm{Ll}_{0}$ puis de nouveau L12) apparaître, avec un domaine d'existence de plus en plus long au fur et à mesure que la dissolution avance (et donc que la concentration en Pd diminue). Les régimes de variation rapide de la concentration d'un sous-réseau correspondent au passage des parois d'interphase à la surface.

L'épaisseur critique, à partir de laquelle on voit émerger de l'ordre en surface, résulte d'une compétition entre la cinétique de dissolution des couches déposées et celle de mise en ordre au niveau de l'interface. A la température étudiée, nous avons obtenu que l'épaisseur critique est de $5 \mathrm{MC}$, épaisseur à partir de laquelle la phase $\mathrm{Ll}_{2}$ (pauvre en $\mathrm{Pd}$ ) apparaît en surface.

Rappelons que le profil de concentration obtenu lors de la dissolution du dépôt d'une monocouche (figure 1) a certaines caractéristiques d'un profil de concentration d'équilibre (appauvrissement du plan de surface en Pd et amortissement oscillant des concentrations). On peut dès lors s'interroger sur le lien entre la surface des phases ordonnées apparaissant au cours de la dissolution du dépôt de $10 \mathrm{MC}$ (communément appelées alliages de surface) et les surfaces des composés $\mathrm{Pd}_{c} \mathrm{Cu}_{1-c}$ à l'équilibre (autrement dit les surfaces d'alliage). Le concept d'équilibre local [9], exposé dans la section suivante, permet de définir rigoureusement la nature de ce lien.

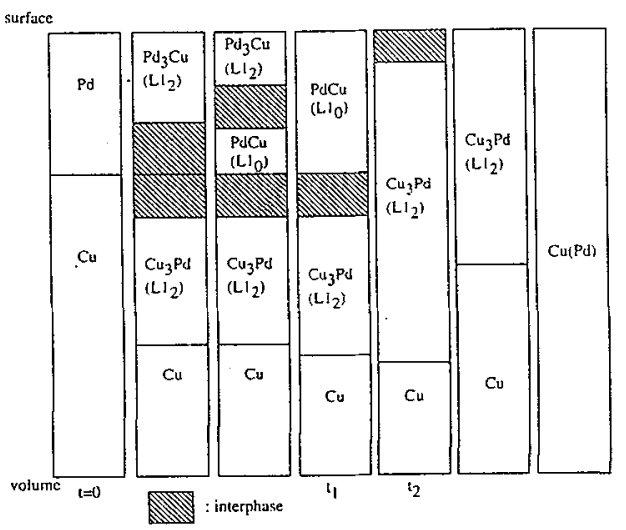

Figure 3: Schéma représentant l'apparition et la disparition spatio-temporelle des phases ordonnées lors d'un dépôt de $10 \mathrm{MC} \mathrm{Pd} / \mathrm{Cu}(111)$ à $\mathrm{T} / \mathrm{T}_{\mathrm{C}}=0,82$.

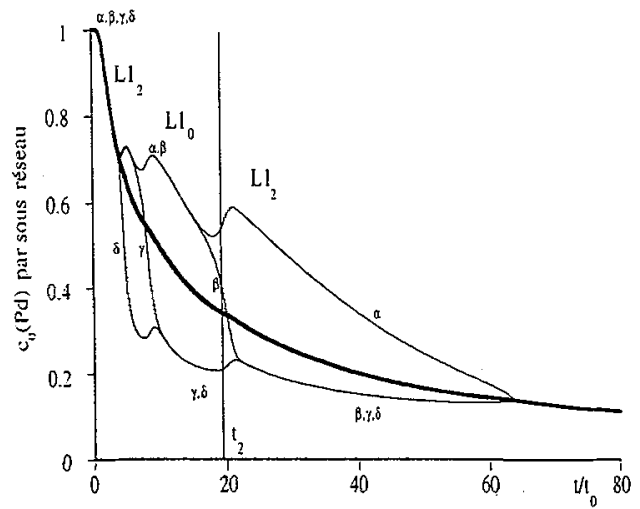

Figure 4: Évolution temporelle des concentrations par sousréseau du plan de surface, $\mathrm{c}_{0}^{\mathrm{i}}(\mathrm{t})$ pour la dissolution de $10 \mathrm{MC}$ $\mathrm{Pd} / \mathrm{Cu}(111)$ à $\mathrm{T} / \mathrm{Tc}=0,82$. La courbe en trait épais représente la concentration moyenne du plan de surface $\mathrm{co}$.

\section{EQUILIBRE LOCAL}

A temps infini, la cinétique de dissolution conduit à l'état d'équilibre, en l'occurrence à une solution solide infiniment diluée, caractérisée par un potentiel chimique homogène. A l'inverse l'état initial est caractérisé par un très fort gradient de potentiel chimique à l'interface initiale et à la surface, les deux lieux étant confondus pour un dépôt de $1 \mathrm{MC}$. La force motrice de la dissolution étant la réduction de ces gradients, les cinétiques seront rapides dans ces configurations, comme en témoigne le stade initial observé lors de la dissolution d'une monocouche. De plus, à cause de l'inhomogénéité due à la surface, les fréquences de saut entre les premiers plans seront plus élevées que dans le reste du cristal, ce qui offre la possibilité d'être "à l'équilibre" sur une certaine profondeur, autrement dit d'avoir un potentiel chimique constant sur cette épaisseur. Cette notion d'équilibre local [9] peut se définir rigoureusement de la façon suivante:

Une région de p plans atomiques sera dite à l'équilibre local à l'instant t, si le profil cinétique à cet instant correspond au profil minimisant l'énergie libre d'une couche mince de même concentration moyenne, avec comme conditions aux limites la donnée de la concentration du plan limitant cette région pour le profil cinétique à l'instant $t$.

La manière la plus directe pour déterminer l'extension spatiale de l'équilibre local est de considérer le potentiel chimique par plan [10]. La zone où celui-ci est constant déterminera la portée de l'équilibre local. Ceci est illustré figure 2, qui montre pour l'instant $\mathrm{t} 1$ (figure 1) de la cinétique de dissolution de $1 \mathrm{MC}$ de $\mathrm{Pd} / \mathrm{Cu}(111)$ le potentiel chimique par plan, ainsi que le profil cinétique et les profils d'équilibre pour différentes épaisseurs. 
Il apparaît clairement, tant en considérant le potentiel chimique que la reconstitution des profils d'équilibre, que les trois premiers plans $\left(c_{0}, c_{1}, c_{2}\right)$ sont à l'équilibre local. Au-delà, l'équilibre local n'est plus valable, comme en témoigne l'écart entre les profils cinétiques et d'équilibre pour une reconstitution sur 8 plans (figure 2). Remarquons que l'extension spatiale de l'équilibre local correspond à la transition observée dans le profil cinétique entre la région où les concentrations oscillent plan par plan (ce qui est typique d'un profil de concentration d'équilibre pour un alliage à tendance à l'ordre comme $\mathrm{Cu}-\mathrm{Pd}$ ) et la région où le profil de concentration est monotone (caractéristique d'un profil de diffusion de type Fickien). La dissolution fait ainsi apparaître deux longueurs caractéristiques:

- La première est la longueur de corrélation, $\xi$, qui caractérise, à l'équilibre, l'amortissement d'une fluctuation de concentration [5]. Dans le cas présent, elle indique le nombre de plans affectés par la ségrégation superficielle et ainsi la portée possible de l'équilibre local. Pour l'exemple de la figure 2 , le calcul conduit à une longueur de $0,7 \mathrm{~d}$ (où $\mathrm{d}$ est la distance interplanaire). Ainsi, sur une profondeur de l'ordre de $3 \xi$, l'équilibre local est vérifié et se caractérise par des oscillations de concentration.

- La deuxième est la longueur de diffusion, l, égale à $\sqrt{ } 4 \mathrm{Dt}$. Elle indique l'épaisseur touchée par la diffusion. Ainsi, pour une profondeur comprise entre $\xi$ et $l$, la nature du profil de concentration n'est plus liée à des considérations d'équilibre, mais de cinétique, ce qui explique son caractère monotone.

Nous avons vérifié de la même façon que, pour le dépôt de $10 \mathrm{MC}$, l'équilibre local de surface est vérifié sur une profondeur similaire, tant pour la concentration moyenne que pour le paramètre d'ordre. Inversement, il n'est pas vérifié en général pour les parois d'interphase, le gradient de concentration (relié à la largeur du biphasage entre les phases à l'équilibre) étant trop faible pour imposer cet équilibre local. Cependant, quand la paroi d'interphase remonte à la surface (par exemple, interphase $\mathrm{L} 1_{0} / \mathrm{L} 1_{2}$ à l'instant $\mathrm{t}_{2}$ sur la figure 4), l'effet de surface est suffisamment fort pour imposer de nouveau l'équilibre local, même sur le paramètre d'ordre, ce qui n'était pas évident au vu de la variation rapide de la concentration du sousréseau $\beta$ en surface.

\section{CONCLUSION}

A l'aide du modèle cinétique KTBIM, nous avons obtenu les cinétiques de dissolution de dépôts d'épaisseur variable de $\mathrm{Pd} / \mathrm{Cu}$ (111). Deux régimes cinétiques ont été mis en évidence : un régime initial de dissolution rapide suivi d'un régime plus lent. Au-delà d'une épaisseur de dépôt de $5 \mathrm{MC}$, nous obtenons une succession spatio-temporelle d'alliages de surface ordonnés.

Le concept d'équilibre local permet de comprendre l'origine de la nature du profil de concentration au cours de la dissolution. Proche de la surface, sur une épaisseur caractérisée par la longueur de corrélation, le profil cinétique de concentration obéit à l'équilibre local et est donc oscillant pour un alliage à tendance à l'ordre. Au-delà, sur une profondeur caractérisée par la longueur de diffusion, le profil cinétique est très proche de celui obtenu par diffusion Fickienne, au moins pour le dépôt d'l MC. Pour les dépôts plus épais, les parois d'interphase séparant les différentes phases ordonnées ne sont pas à l'équilibre local, sauf quand elles débouchent à la surface.

Ce type d'approche sera étendu à des cas où des cinétiques de dissolution ont été étudiées expérimentalement, à savoir $\mathrm{Pd} / \mathrm{Cu}$ (100) [3] et $\mathrm{Sn} / \mathrm{Pt}$ (111) [11].

\section{Références}

[1] Campbell C.T., Annu. Rev. Phys. Chem. 41 (1990) 775

[2] Senhaji A., Tréglia G., Legrand B., Barrett N.T., Guillot C., Villette B., Surf. Sci. 274 (1992) 297

[3] Pope T.D., Griffiths K., Zhdanov V.P., Norton P.R., Phys. Rev. B 50 (1994) 18553 (et références citées dans cet article)

[4] Tréglia G., Legrand B., Ducastelle F., Europhys. Lett. 7 (1988) 575

[5] Ducastelle F., Order and Phase Stability in Alloys (North-Holland, Amsterdam, 1991)

[6] Gallis C., Legrand B., Saùl A., Tréglia G., Hecquet P., Salanon B., Surf. Sci. 352-354 (1996) 588

[7] Diffusion in Solid Metals and Alloys, Landolt-Börnstein vol. 26 (Springer Verlag, Berlin, 1990)

[8] d'Heurle F.M., Reactive Phase Formation at Interfaces and Diffusion Processes, Y. Limoge et J.L. Bocquet, Eds. (Trans Tech Publications, Suisse, 1994) p. 1

[9] Lagües M., Domange J.L., Surf. Sci. 47 (1975) 77

[10] Martin G., Phys. Rev. B 41 (1990) 2279

[11] Atrei A., Bardi U., Wu J.X., Zanazzi E., Rovida G., Surf. Sci. 290 (1993) 286 